\title{
Prediction and prognostic significance of BCAR3 expression in patients with multiple myeloma
}

\author{
Weilong Zhang ${ }^{1 \dagger}$, Yuansheng $\mathrm{Lin}^{2 \dagger}{ }^{+}$, Xiaoni $\mathrm{Liu}^{3 \dagger}{ }^{+}$Xue He${ }^{4}$, Ye Zhang ${ }^{5}$, Wei Fu ${ }^{6}$, Zuozhen Yang ${ }^{1}$, Ping Yang ${ }^{1}$,
} Jing Wang ${ }^{1}$, Kai Hu${ }^{1}$, Xiuru Zhang ${ }^{4}$, Weiyou Liu $^{3^{*}}$, Xiaoliang Yuan ${ }^{3^{*}}$ and Hongmei Jing ${ }^{1 *}$

\begin{abstract}
Background: Multiple myeloma (MM) is the plasma cell tumor, which is characterized by clonal proliferation of tumor cells, with high risk of progression to renal impairment, bone damage and amyloidosis. Although the survival rate of patients with MM has improved in the past decade, most people inevitably relapse. The treatment and prognosis of $\mathrm{MM}$ are still urgent problems. Breast Cancer Antiestrogen Resistance 3 (BCAR3) is a protein-coding gene that is associated with many tumors. However, there have been few studies on the relationship of BCAR3 and MM.

Methods: We analyzed 1878 MM patients (1930 samples) from 7 independent datasets. First, we compared the BCAR3 expression level of MM patients in different stages and MM patients with different amplification of 1q21. Second, we analyzed BCAR3 expression levels in MM patients with different molecular subtypes. Finally, we explored the event-free survival rate (EFS) and overall survival rate (OS) of MM patients with high or low BCAR3 expression, including patients before and after relapse, and their therapeutic responses to bortezomib and dexamethasone.

Results: The expression of BCAR3 showed a decreasing trend in stages I, II and III ( $P=0.00068)$. With the increase of $1 \mathrm{q} 21$ amplification level, the expression of BCAR3 decreased $(P=0.022)$. Patients with high BCAR3 expression had higher EFS and OS (EFS: $P<0.0001$, OS: $P<0.0001$ ). The expression of BCAR3 gene before relapse was higher than that after relapse $(P=0.0045)$. $B C A R 3$ is an independent factor affecting prognosis ( $E F S: P=5.17 E-03 ; O S: P=3.33 E-04)$.

Conclusion: We found that high expression level of BCAR3 predicted better prognosis of MM patients. Low expression of BCAR3 at diagnosis can predict early relapse. BCAR3 is an independent prognostic factor for MM. BCAR3 can be used as a potential biomarker.
\end{abstract}

Keywords: BCAR3, Multiple myeloma, Prognosis, Gene expression profile

\section{Background}

MM is a B cell differentiated tumor characterized by clonal proliferation of tumor cells [1-3]. MM is a

\footnotetext{
*Correspondence: gzweiyou@163.com; yxlyyxs@126.com; drjinghm@163.com

${ }^{\dagger}$ Weilong Zhang, Yuansheng Lin and Xiaoni Liu contributed equally to this work

${ }^{1}$ Department of Hematology, Lymphoma Research Center, Peking University Third Hospital, No. 49 North Garden Road, Haidian District, Beijing 100191, People's Republic of China

${ }^{3}$ Department of Respiratory Medicine, The First Affiliated Hospital of Gannan Medical University, No. 23 Qingnian Road, Zhanggong District, Ganzhou 341000, People's Republic of China

Full list of author information is available at the end of the article
}

heterogeneous disease with different clinical characteristics [4]. By recognizing genetic mechanism and mutation, a normal plasma cell transited into the following disease stages: monoclonal gammopathy of undetermined significance, smouldering myeloma, myeloma and plasma cell leukaemia [5]. The International Staging System (ISS) uses the staging criteria to divide MM into three phases, combining serum albumin levels with $\beta 2$-microglobulin to determine the prognosis of MM patients [6]. Revised International Staging System (R-ISS) is a simple and effective prognostic staging system that combines three prognostic tools (ISS: International Staging System, CA: chromosomal abnormalities, LDH: lactate

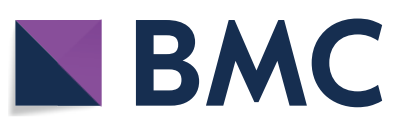

(c) The Author(s) 2018. This article is distributed under the terms of the Creative Commons Attribution 4.0 International License (http://creativecommons.org/licenses/by/4.0/), which permits unrestricted use, distribution, and reproduction in any medium, provided you give appropriate credit to the original author(s) and the source, provide a link to the Creative Commons license, and indicate if changes were made. The Creative Commons Public Domain Dedication waiver (http://creativecommons.org/ publicdomain/zero/1.0/) applies to the data made available in this article, unless otherwise stated. 
dehydrogenase) to better assess patient prognosis [7]. Gene expression profiling (GEP) is important for revealing MM molecular heterogeneity of different patients. The molecular classification established by UAMS (The University of Arkansas for Medical Sciences) based on GEP data [8]. The molecular basis of MM is defined by unsupervised clustering analysis of mRNA expression profiles, which are divided into seven molecular subtypes [9]. Recurrence is a major problem in most MM patients. Among all MM patients, early recurrence and poor prognosis are about $10 \%$ to $15 \%$ [10].

1q21 amplification is the most common chromosomal aberration in $\mathrm{MM}$ and is considered a high-risk genetic feature [11]. The function of the BCAR3 (Breast Cancer Antiestrogen Resistance 3) gene is varied. BCAR3 can promote cell migration, proliferation, and BCAR3 was identified as a molecular linkage between PTP and Cas [12-14]. BCAR3 protein participates in the signaling pathway of EGF through its SH2 domain, leading to cell cycle progression, and BCAR3 itself belong to a mitogenic signaling pathway $[15,16]$. BCAR3 and p130Cas were associated with anti-estrogen in breast cancer, Rac activation. BCAR3 can regulate the Src signal transmission of BCAR3-p130 (cas) complex dependence mode [17-19]. During the initial stage of gonadal development, BCAR3 in gonad development is very important and is expressed in XY gonads [20]. Studies have shown that the BCAR3 gene is the first spontaneous mutation associated with cataracts caused by lens compression. This new cataract model could provide further knowledge about the function of BCAR3 protein [21]. In the study of tamoxifen in the treatment of metastatic breast cancer, high expression of BCAR3 is related to good progression-free survival, and the expression level of BCAR3 in primary breast tumors is relatively low, which is related to the survival rate of distant metastasis [14]. Ovarian cancer is a disease characterized by tumor heterogeneity, which is difficult to be diagnosed and treated. Studies have shown that inhibiting the expression of BCAR3 gene can inhibit the cell proliferation of ovarian cancer [22]. However, there is no study reporting the relationship between BCAR3 and MM so far. By integrating data of 1878 MM patients, we found that BCAR3 gene is closely related to MM.

\section{Methods}

\section{Data source}

In our study, gene expression microarrays of 1878 MM patients (1930 samples) were derived from Gene Expression Omnibus database, including datasets GSE24080 (559 samples) [23], GSE82307 (66 samples) [24], GSE19784 (308 samples) [25], GSE83503 (585 samples) [26], GSE9782 (238 samples) [27], GSE39754 (136 samples) and GSE19554 (38 samples) [28, 29]. The criteria for patient selection in our research were stated. 1) All MM patients with the published high throughput gene expression data. 2) All the patients should have some information such as clinical features, biochemical examination, karyotype, therapy or therapy response. The study was approved by the Human Research Ethics Committee of Peking University third hospital. The research was conducted in accordance International Conference on and the Declaration of Helsinki.

\section{Microarray analysis}

All microarray data were analyzed, and the significantly abnormal expression genes were systematically screened as predictive biomarkers. The different expression of genes between BCAR3-low to BCAR3high group were also analyzed and ranked by foldchange values $(\log 2, \mathrm{FC}>0.8$ or $<-0.8, \mathrm{P}<0.05)$.

We retrieved GSE24080 (559 samples) from the NCBI GEO database and analyzed the expression of BCAR3 among different ISS stages, different molecular subtypes, different 1q21 amplification levels, and different survival (EFS and OS). We retrieved GSE82307 (66 samples) from the NCBI GEO database. We analyzed the expression of BCAR3 before and after recurrence in the same patient. We retrieved GSE19554 (38 samples) in $19 \mathrm{MM}$ patients from the NCBI GEO database, and we analyzed BCAR3 expression between the baseline (before chemotherapy) and pre-1st (after induction of chemotherapy, before bone marrow transplantation) in the same sample. We retrieved GSE19784 (308 samples) from the NCBI GEO database and analyzed the expression of BCAR3 in different molecular subtypes. We retrieved GSE83503 (585 samples) from the NCBI GEO database and analyzed the expression of BCAR3 in the recurrence and non-recurrence groups. We retrieved GSE9782 (238 samples) from the NCBI GEO database and analyzed the expression of BCAR3 between the different treatment response of bortezomib and dexamethasone. All patients from GSE39754 (136 samples) were treated with vincristine, adriamycin, and dexamethasone (VAD), followed by autologous stem cell transplantation (ASCT). Then measuring "therapeutic response" after ASCT. Analysis of BCAR3 expression in each treatment response compared to the average of all treatment responses. Therapeutic response: complete response (CR), very good partial response (VGPR), partial response (PR), no response, stable disease (NR), no response, progressive disease (Prog).

\section{Gene ontology (GO) analysis}

Use the DAVID to analyze the 559 samples (dataset GSE24080), and find out the enrichment pathways for 
different expressed genes between BCAR3-low and BCAR3-high group [30]. The results were ranked by the $\mathrm{P}$ value $(-\log 10, \mathrm{P}<0.05)$.

\section{Statistical analyses}

Statistical analyses were performed by R software v3.1.3 (ggplot2 and survminer package). The log-rank test and cox regression multivariate analysis were used for survival analysis. $\mathrm{P}$ value $<0.05$ was considered statistically significant.

\section{Result}

BCAR3 is expressed in multiple myeloma of different molecular types

We compared BCAR3 gene expression level of different 1q21 amplification. With the increase of the amplification level of 1q21, the expression level of BCAR3 showed an overall downward trend (Fig. 1a, $\mathrm{P}=0.022$, Kruskal-Wallis test). New UAMS classifies MM into seven subtypes based on different gene expression profiles. Comparison of expression levels in seven molecular types showed that the expression level of BCAR3 gene is different in molecular subtypes. CD2 subtype had the highest BCAR3 expression, while the proliferation (PR) subtype and MAF subtype had the lowest BCAR3 expression (Fig. 1b,

\section{type 审 2 宊 3 审 4+}
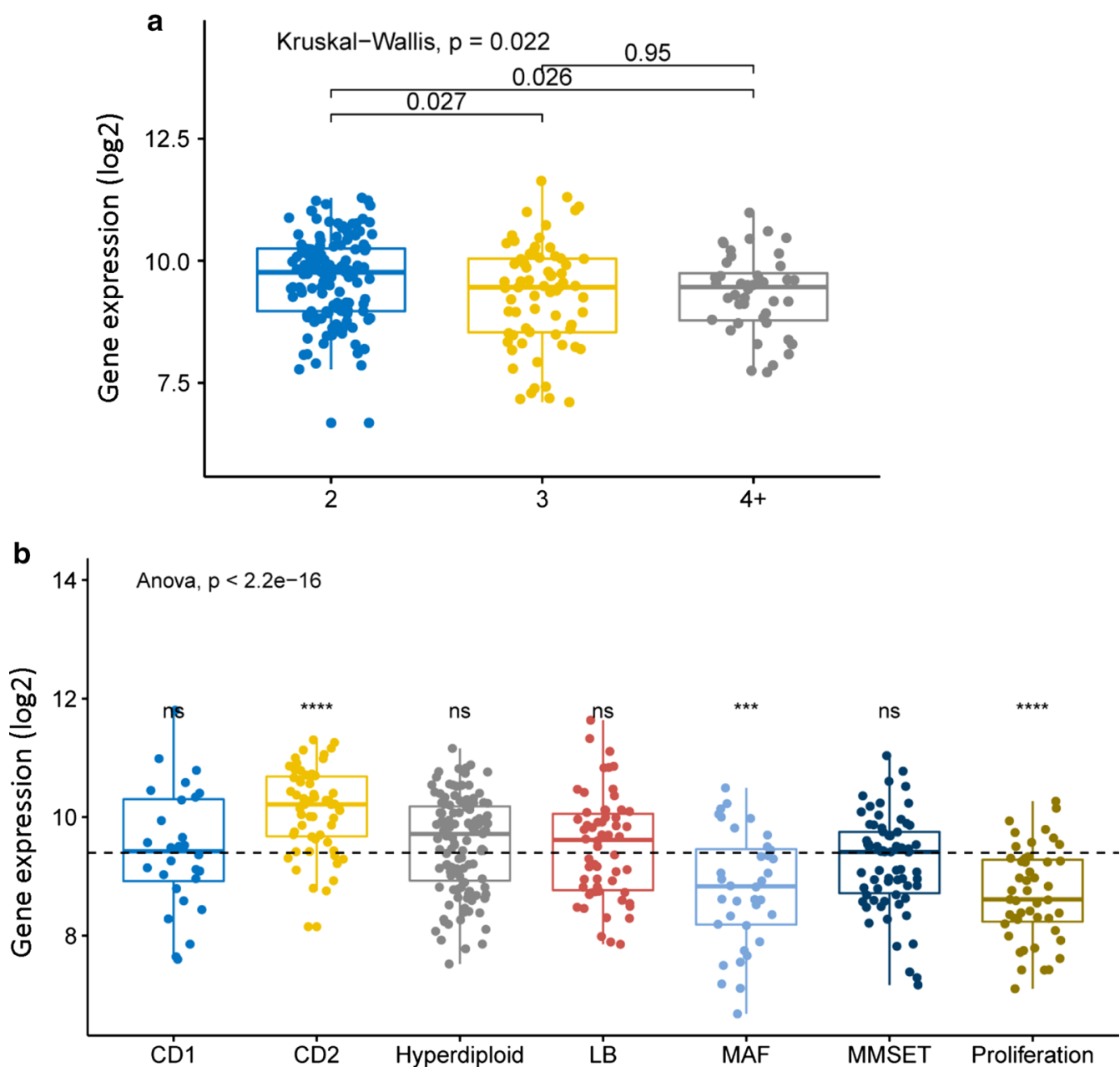

Fig. 1 The expression of BCAR3 in different amplification levels of $1 \mathrm{q} 21$ and 7 molecular subtypes. a BCAR3 expression levels at different amplification levels of 1q21. The X-axis represents the $1 \mathrm{q} 21$ amplification, the $Y$-axis represent BCAR3 expression level. $P=0.022$, Kruskal-Wallis test. b Comparison of BCAR3 expression levels in 7 molecular subtypes. The $Y$-axis represents BCAR3 expression levels; the $X$-axis represents 7 molecular subtypes. $\mathrm{P}<2.2 \mathrm{E}-16$, Anova test. We use statistically significant symbol: $n s ; \mathrm{P}>0.05$; ${ }^{*} \mathrm{P}<=0.05$; ${ }^{*} \mathrm{P}<=0.01$; ${ }^{*} \mathrm{P}<=0.001$; ****P<=0.0001 


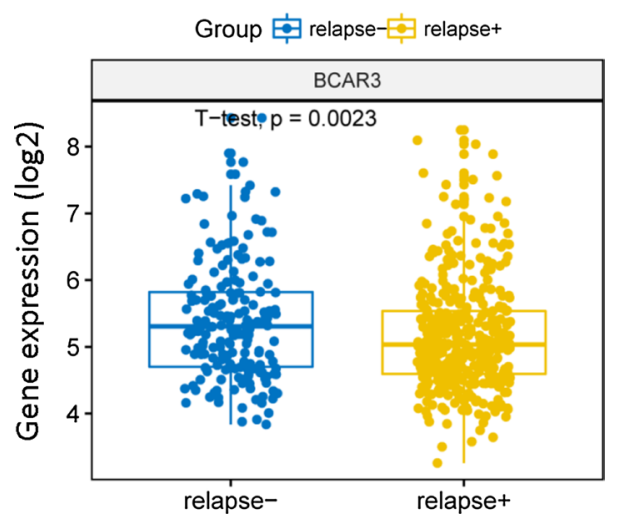

Fig. 2 The expression level of BCAR3 in the relapse group and the non-relapse group from dataset GSE83503 (585 samples). The blue one represents non-relapse group and the yellow one represents relapse group. $P=0.0023$, Unpaired t test, two sided

$\mathrm{P}<2.2 \mathrm{E}-16$, Anova test). We also analyzed the dataset GSE19784, a total of 308 samples, the expression level of BCAR3 in CD1 subtype and CD2 subtype was the highest, while that of PR (proliferation) subtype was the lowest (Additional file 1: Figure S1, $\mathrm{P}=7.8 \mathrm{E}-10$, Anova test).

\section{Relapse is associated with low BCAR3 expression}

The expression of BCAR3 gene was statistically different in the non-relapse group and the relapse group of MM patients in dataset GSE83503 (585 samples), and the expression in the non-relapse group was significantly higher than that in the relapse group (Fig. 2, $P=0.0023$, Unpaired t test, two sided). Therefore low expression of BCAR3 at diagnosis can predict early relapse.

The expression of BCAR3 gene before (at diagnosis) and after relapse (in remission) was compared in dataset GSE82307 (66 samples). The expression of BCAR3 gene in the same MM patients before and after relapse was statistically different, and the expression before relapse was significantly higher than that after recurrence (Fig. 3a, P $=0.0045$, Wilcoxon test). However, the expression of BCAR3 gene was not changed before and after chemotherapy in the same patient (Fig. 3b, $\mathrm{P}=0.39$, Wilcoxon test).

\section{BCAR3 gene can predict survival of MM}

Patients with high BCAR3 expression had higher EFS and OS, while low BCAR3 expression had lower EFS and OS in dataset GES24080 (559 samples) (Fig. 4a, EFS: $\mathrm{P}<0.0001$, OS: $\mathrm{P}<0.0001$, Log-rank test). In the survival curve of ISS stage I patients, we can see that patients of ISS stage I have higher EFS and OS when BCAR3 gene is highly expressed (Fig. 4b, EFS: $\mathrm{P}=0.013$, OS: $\mathrm{P}=0.0013$, Log-rank test). Patients of ISS stage II and III also have higher EFS and OS when BCAR3 gene is highly expressed (Fig. 4c, EFS: $\mathrm{P}=0.002$, OS: $\mathrm{P}=1 \mathrm{E}-04$, Log-rank test).
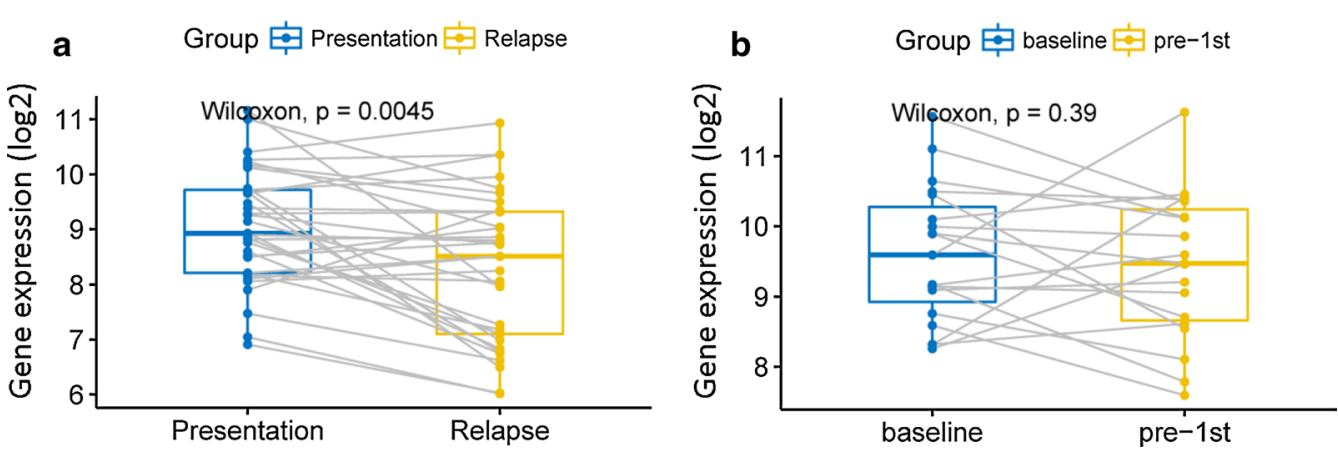

Fig. 3 We compared BCAR3 gene expression before and after therapy. Gray lines represent a trend in the same indicator for a same patient. a BCAR3 expression levels were compared before and after relapse in the same patient. $P=0.0045$, Wilcoxon test. $\mathbf{b}$ The expression levels of BCAR3 baseline and pre-1st were compared. $\mathrm{P}=0.39$, Wilcoxon test

(See figure on next page.)

Fig. 4 The survival of EFS and OS in BCAR3-low and BCAR3-high group. The X-axis represents the EFS/OS time (months), the Y-axis represents the survival probability. Yellow line represents BCAR3-low group, blue line represents BCAR3-high group. EFS: event-free survival rate; OS: overall survival rate. a The survival of EFS and OS in BCAR3-low and BCAR3-high group from 559 patients. EFS: $P<0.0001, O S: P<0.0001$, Log-rank test. b Survival curve in patients with ISS stage I. EFS: $P=0.013, \mathrm{OS}: \mathrm{P}=0.0013$, Log-rank test. $\mathbf{c}$ Survival curve in patients with ISS stage II or III between BCAR3-high and BCAR3-low group. The X-axis represents the EFS/OS time (months); the $Y$-axis represents the survival probability. $E F S: P=0.002, O S$ : $P=1 E-04$, Log-rank test 

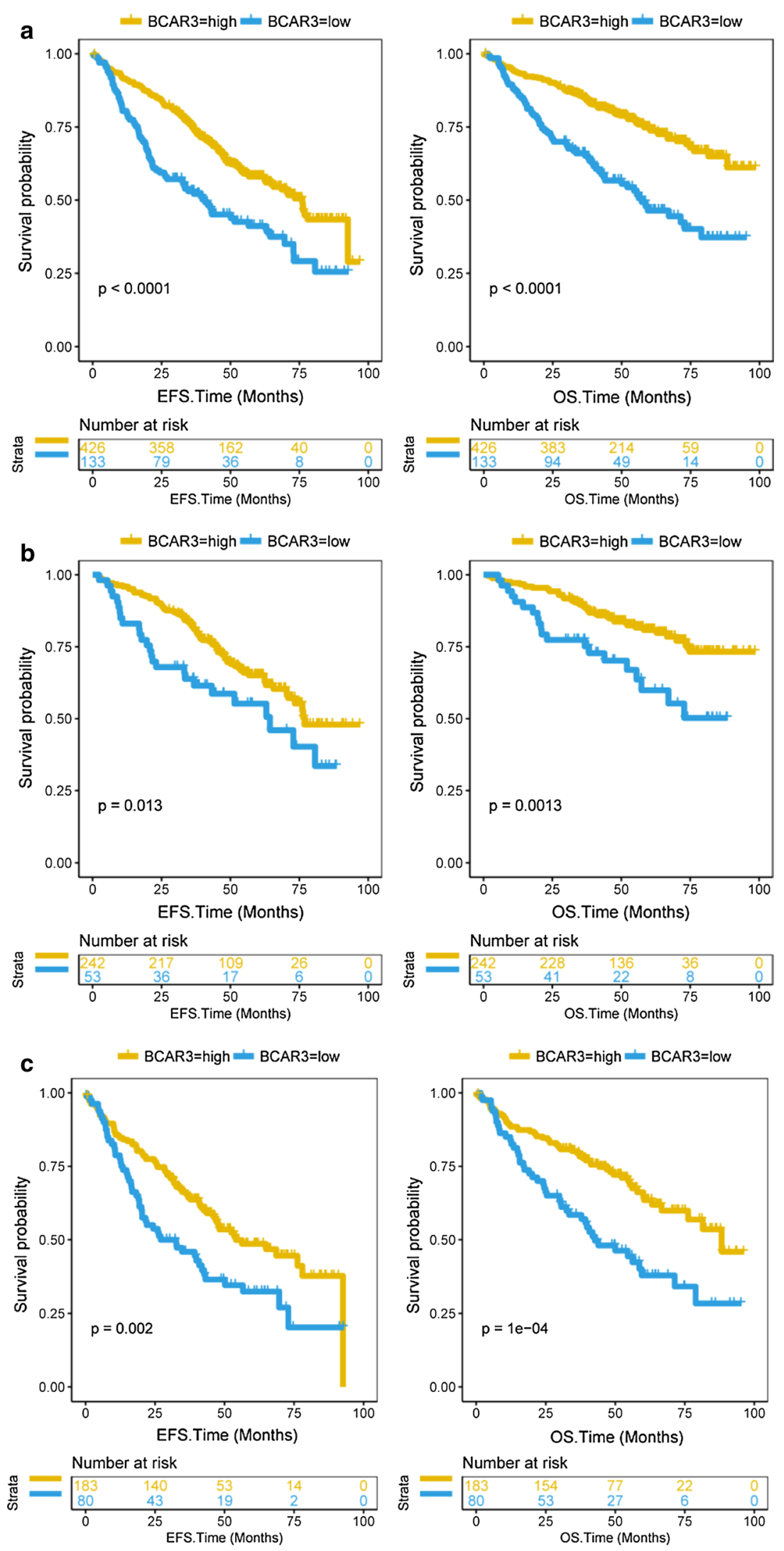


\section{BCAR3 higher expressed in stage I than stage II and III} in multiple myeloma

The expression levels of BCAR3 in GSE24080 dataset (559 samples) were compared at different ISS stages. The expression of BCAR3 showed a decreasing trend in stages I, II and III (Additional file 1: Figure S2A, P $=0.00068$, Kruskal-Wallis test). There was no statistically significant difference between stage II and III (Additional file 1: Figure S2A, $\mathrm{P}=0.39$, Wilcoxon test). In addition, the expression levels of BCAR3 under different serotype stratification were compared at different stages. In serum immunoglobulin A (IgA) group and serum immunoglobulin $\mathrm{G}$ (IgG) group, the expression of BCAR3 in stages I, II and III decreased gradually (Additional file 1: Figure S2B, IgA: $\mathrm{P}=0.013$; IgG: $\mathrm{P}=0.04$; Kruskal-Wallis test). However, there was no statistical significance in the serum free light chain (FLC, light chain myeloma subtype) group (Additional file 1: Figure S2B, FLC: $\mathrm{P}=0.39$; Kruskal-Wallis test).

\section{The BCAR3 gene is associated with immunity related pathway}

68 up-regulated and 23 down-regulated genes were found between the BCAR3-high and BCAR3-low group. Heat map shows top 12 up-regulated genes and top 12 downregulated genes (Additional file 1: Figure S3A, $\mathrm{P}<0.01$ ). Among the enriched pathway of different expressed gene, immune response pathway (GO: 0006955) and B cell receptor signaling pathway (GO: 0050853) are the most related (Additional file 1: Figure $\mathrm{S} 3 \mathrm{~B}, \mathrm{P}<0.01$ ). In the immune response pathway, all the 11 different expressed genes such as CCL18, CD27, CD74, CTSW and CXCL12 were up-regulated in the BCAR3-high group compared with the BCAR3-low group (Additional file 1: Figure S4, $\mathrm{P}<0.001$, Unpaired $\mathrm{t}$ test, two sided).

\section{Comparison of BCAR3 expression of different therapeutic responses to bortezomib and dexamethasone}

BCAR3 expression of different therapeutic responses to bortezomib and dexamethasone was compared in 238 samples of GSE9782 dataset. There was no difference in the expression of the BCAR3 in the post-treatment responses of bortezomib and dexamethasone (Additional file 1: Figure S5, bortezomib: $\mathrm{P}=0.21$ dexamethasone: $\mathrm{P}=0.65$, Anova test). There was also no significant difference in the expression of BCAR3 in each treatment response in $136 \mathrm{MM}$ patients from GSE39754 dataset (Additional file 1: Figure S6, $\mathrm{P}=0.96$, Anova test).

BCAR3 is an independent factor affecting prognosis of MM Cox regression multivariate analysis was used to compare the clinical characteristics of 559 patients with
MM. In the EFS, B2M $(\mathrm{HR}=1.38,95 \%$ CI $1.01-1.88$; $\mathrm{P}=4.22 \mathrm{E}-02), \quad \mathrm{MRI} \quad(\mathrm{HR}=1.40,95 \%$ CI $1.07-1.84$; $\mathrm{P}=1.47 \mathrm{E}-02), \mathrm{BMPC}(\mathrm{HR}=1.39,95 \%$ CI $1.01-1.92$; $\mathrm{P}=4.56 \mathrm{E}-02)$ and BCAR3 ( $\mathrm{HR}=0.66$, 95\% CI 0.5-0.88; $\mathrm{P}=5.17 \mathrm{E}-03)$ are prognostic factors for MM. BCAR3 $(\mathrm{P}=5.17 \mathrm{E}-03)$ is a prognostic factor independent of B2M, MRI, and BMPC in myeloma patients (Additional file 2: table S1). In the OS, B2M ( $\mathrm{HR}=1.59,95 \%$ CI $1.09-2.33 ; \mathrm{P}=1.74 \mathrm{E}-02)$, MRI $(\mathrm{HR}=1.80,95 \% \mathrm{CI}$ $1.28-2.52 ; \mathrm{P}=7.52 \mathrm{E}-04)$, and BCAR3 $(\mathrm{HR}=0.55,95 \%$ CI $0.39-0.76 ; \mathrm{P}=3.33 \mathrm{E}-04)$ are prognostic factors for MM (Additional file 2: Table S1). BCAR3 is the most statistically significant biomarker of all variable that we analyzed in both EFS and in OS (Additional file 2: Table S1, EFS: $\mathrm{P}=5.17 \mathrm{E}-03$, OS: $\mathrm{P}=3.33 \mathrm{E}-04$, Cox regression multivariate analysis). BCAR3 is an independent factor in the prognosis of MM.

We also analyzed whether the clinical characteristics were significantly different between BCAR3-low group (133 samples) and BCAR3-high group (426 samples). Age $(P=0.219)$, sex $(P=0.733)$, race $(P=0.937)$ and isotypes $(\mathrm{P}=0.414)$ were not statistically significant (Additional file 2: Table $\mathrm{S} 2$, Fisher's exact test). B2M ( $\mathrm{P}=0.004)$, LDH $(\mathrm{P}=0.007)$, HGB $(\mathrm{P}=0.038)$, ASPC $(\mathrm{P}=0.031)$, BMPC $(\mathrm{P}<0.001)$ and MRI $(\mathrm{P}=0.001)$ are significantly different in BCAR3-low group and BCAR3-high group (Additional file 2: Table S2, Unpaired $\mathrm{t}$ test, two sided). CRP $(\mathrm{P}=0.738)$, CRECT $(\mathrm{P}=0.426)$ and ALB $(\mathrm{P}=0.082)$ were not significantly different in BCAR3-low group and BCAR3-high group (Additional file 2: Table S2, Unpaired $\mathrm{t}$ test, two sided).

\section{Discussion}

For MM patients with age less than 65 years, high-dose treatment with autologous stem cells is a first-line effective treatment [31]. But MM is a kind of incurable disease and a B-cell differentiated tumor, whose character is cloned plasma cell proliferations, kidney failure, anemia, dissolve the bony lesions, hypercalcemia and infection [32-34]. Free monoclonal immunoglobulins in serum or urine were found to be related with malignant plasma cell marrow infiltration $[35,36]$. BCAR3 is a protein-coding gene, diseases associated with BCAR3 include estrogen resistance, breast cancer, and cataracts. However, the relationship between BCAR3 and MM has not been studied. Therefore, we analyzed the expression level of BCAR3 gene in patients with MM, and found that high BCAR3 expression has better prognosis.

Different mechanisms for BCAR3 in cancer were reported. (1) The interaction between BCAR3 and cas is blocked by the L744E/R748E mutation of BCAR3, the activity of Rac1 is decreased, and the tension of RhoA may be increased, which provides stable adhesion and 
slows disassembly, thus inhibiting tumor progression. In contrast, BCAR3 and cas interact to activate Rac1, rapidly breaking down adhesion and resulting tumor invasion [16]. (2) High expression of AND-34/BCAR3 activates the activity of Rac and Pak1, thereby activating the CyclinD1 promoter, making anti-estrogen resistance and progression of breast cancer cells. In contrast, a dominant negative for Rac and Pak1 inhibits the activation of the CyclinD1 promoter [37]. (3) It is believed that breast tumors initially depend on estrogen for development. Through experiments, BCAR3 is closely related to in vitro anti-estrogen resistance. There is an inverse relationship between BCAR3 expression and ER (estrogen receptor) protein expression in breast and ovarian cells. Tumor cell growth inhibition and hormonal signal blockade are due to the competition of ER by antiestrogens. When BCAR3 is low expressed, ER is highly expressed and inhibits tumor progression [38]. (4) TGF- $\beta / S m a d$ is a signaling pathway of BCAR3 in breast invasive tumors, and BCAR3 inhibits the conduction of TGF $\beta /$ Smad signaling when highly expressed, thereby inhibiting tumor progression [14]. So, overexpression of BCAR3 in breast cancer cells can promote cell migration and invasion in most researches. However, in some researches, overexpression of BCAR3 in breast cancer cells can inhibit cell migration and invasion. In our research, we found that BCAR3 is closely related to the immune response pathway (Additional file 1: Figure S3B). All the 11 different expressed genes such as CCL18, CD27, CD74, CTSW and CXCL12 were up-regulated in the BCAR3-high group compared with the BCAR3-low group, which suggest that in the immune response pathway was activated (Additional file 1: Figure S4). For example, CXCL12 is a gene in the immune response pathway. The expression of CXCL12 inhibits the metastasis and growth of primary breast cancer [39]. So, high expression of BCAR3 may inhibit MM growth through up-regulated genes in the immune response pathway.

High dose autologous bone marrow transplantation has been reported and achieved good results [40]. Pre-relapse mortality was significantly lower than postrelapse [41]. The survival rate of patients after relapse is reduced. Our study shows that in patients with MM, low expression of BCAR3 at diagnosis can predict early relapse. In addition, $\mathrm{MM}$ is a clonal plasmacytoma that can be judged by monoclonal immunoglobulin (IgA, IgG) or increased light chain levels [42]. The heavy/light chain assay can predict the prognosis and surveille disease in patients with myeloma after treatment [43]. We also studied serum IgA and serum IgG as well as light chain and further analyzed the expression of BCAR3 at different stages of each serotype. In serum FLC, there was no significant regularity in the expression of BCAR3 between stages I, II and III. However, in the serum IgA group and the serum IgG group, the expression of BCAR3 of stage I MM was higher than that of stage II and stage III.

BCAR3 is an independent prognostic factor for MM, which can reflect the survival rate of patients with MM. We have some important evidence to support this view: First, patients with high BCAR3 expression had higher EFS and higher OS. Second, MM patients with BCAR3 had higher expression before relapse and lower expression after relapse. Last, B2M, LDH, HGB, ASPC, BMPC and MRI have significant differences in the BCAR3-low and BCAR3-high groups (Additional file 2: Table S2). It was found that high expression of BCAR3 gene predicted good prognosis.

The study has some shortcomings. For example, the molecular mechanism of BCAR3 gene in MM is not in-depth studied. We can also further study from this aspect. In addition, MM may be further divided into low risk, moderate risk and high risk combined the expression level of BCAR3 and other known biomarkers, which is helpful to evaluate the survival of patients. These shortcomings needs to be further studied.

\section{Conclusions}

In summary, our data show that high expression levels of BCAR3 predict a better prognosis for MM patients. Low expression of BCAR3 at diagnosis can predict early relapse. BCAR3 is an independent prognostic factor for MM. BCAR3 can be used as a potential biomarker.

\section{Additional files}

Additional file 1: Figure S1. The expression level of BCAR3 in different molecular subtypes. The $X$-axis represents the 9 molecular subtypes; the $Y$-axis represents the gene expression. The dotted line represents the average of BCAR3 gene expression levels of all molecular subtypes, including CD1, CD2, CTA, HY, MF, MS, myeloid, NFKB, PR. P =7.8E-10, Anova test. Figure S2. The expression level of BCAR3 in different ISS stages and 3 serotypes in MM patients. A, The expression of BCAR3 was compared between different ISS stages in MM patients. The X-axis represents the ISS stages; the $Y$-axis represents BCAR3 expression level $(\log 2) . P=0.00068$, Kruskal-Wallis test. B, The expression of BCAR3 in different ISS stages were compared under different serotype stratification (FLC: Serum free light chain, IgA: Serum immunoglobulin A, IgG: serum immunoglobulin G). Kruskal-Wallis test. Figure $\mathbf{S}$. Heat map of different expression genes between BCAR3-low and BCAR3-high groups and related enrichment pathways. A, Heat map shows top 12 up-regulated genes and top 12 down-regulated genes. The red represents high expression, the white represents intermediate expression, and the green represents low expression. The foldchange $(\log 2)$ of different expressed genes is ranked, and the corresponding P-value $(-\log 10)$ is on the right in the heat map. B, The enrichment pathways for different expression genes. The $X$-axis represents p-value $(-\log 10)$ and the $Y$-axis represents different enriched pathways. Figure S4. The expression levels of 11 different genes in the immune response pathway in the BCAR3-high group and the BCAR3-low group were compared. Unpaired t test, two sided. Figure S5. BCAR3 expression in different therapeutic response to bortezomib and dexamethasone. The 
left side shows the therapeutic response to bortezomib. The therapeutic response to dexamethasone was shown on the right. The expressions of BCAR3 were compared between complete remission (CR), partial remission $(P R)$, minimal response $(M R)$, no change $(N C)$, and disease progression (DP) group. The dotted line represents the average of BCAR3 gene expression levels in all treatment responses. Bortezomib: $P=0.21$, dexamethasone: $P=0.65$, Anova test, two sided. Statistical significance: $n s: P>0.05 ; *: P<=0.05 ;{ }^{* *}: P<=0.01$; $^{* * *}: \mathrm{P}<=0.001$; $^{* * *}: \mathrm{P}<=0.0001$. Figure S6. Comparison of expression levels of the BCAR3 gene in therapeutic responses. The $X$-axis represents the groups of treatment responses to induction chemotherapy and autologous stem cell transplantation; the $Y$-axis represents the expression of BCAR3. The dotted line represents the average of BCAR3 gene expression levels in all treatment responses. Treatment responses: Complete Response (CR); Very Good Partial Response (VGPR); Partial Response (PR); No Response, Stable disease (NR); No Response, Progressive disease (Prog). $P=0.96$, Anova test. Statistical significance: $n s: P>0.05 *: P<=0.05^{* *}: P<=0.01 * * *: P<=0.001$ ****: $P<=0.0001$

Additional file 2: Table S1. Multivariate analysis of clinical prognostic parameters in 559 multiple myeloma patients (Cox regression multivariate analysis). Table S2. Baseline patient characteristics according to the expression level of BCAR3.

\section{Abbreviations}

MM: multiple myeloma; EFS: event-free survival time, defined from date of registration to the occurrence of death from any cause, disease progression or relapse, or censored at the date of last contact; OS: overall survival time, defined from date of registration to the date of death from any cause or censored at the date of last contact; B2M: beta-2 microglobulin; ALB: albumin; HGB: haemoglobin; MRI: number of magnetic resonance imaging (MRI)defined focal lesions (skull, spine, pelvis); BMPC: bone marrow biopsy plasma cells (\%); HR: hazard ratio; Cl: confidence interval; CRP: C-reactive protein; CREAT: creatinine; ASPC: aspirate plasma cells (\%).

\section{Authors' contributions}

HMJ, XLY, WYL and XRZ conceived the project. WLZ, YSL, XNL, YZ and XH analyzed the data. WLZ, YSL, XNL, XH, YZ, WF, ZZY, PY, JW, KH, XRZ, WYL, XLY and $\mathrm{HMJ}$ contributed towards the interpretation of the data. All authors read and approved the final manuscript.

\section{Author details}

${ }^{1}$ Department of Hematology, Lymphoma Research Center, Peking University Third Hospital, No. 49 North Garden Road, Haidian District, Beijing 100191, People's Republic of China. ${ }^{2}$ Gannan Medical University, Ganzhou 341000 China. ${ }^{3}$ Department of Respiratory Medicine, The First Affiliated Hospital of Gannan Medical University, No. 23 Qingnian Road, Zhanggong District, Ganzhou 341000, People's Republic of China. ${ }^{4}$ Department of Pathology, Beijing Tiantan Hospital Affiliated With Capital Medical University, No. 6 Tiantan Xili, Beijing 100050, China. ${ }^{5}$ Melbourne School of Population and Global Health, The University of Melbourne, Melbourne, VIC 3010, Australia. ${ }^{6}$ Peking University Third Hospital, Beijing 100191, China.

\section{Acknowledgements}

We thank the Datasets GSE24080, GSE82307, GSE19784, GSE83503, GSE9782, GSE39754 and GSE19554 from GEO Database.

\section{Competing interests}

The authors declare that they have no competing interests.

\section{Availability of data and materials}

Not applicable.

\section{Consent for publication}

Not applicable.

\section{Ethical approval and consent to participate}

This study was approved by the Helsinki declaration of 1964 and its subsequent amendments.

\section{Funding}

This work was funded by interdisciplinary medicine Seed Fund of Peking University (BMU2018MB004), Beijing Natural Science Foundation (7132183), China Health Promotion Foundation (CHPF-zlkysx-001).

\section{Publisher's Note}

Springer Nature remains neutral with regard to jurisdictional claims in published maps and institutional affiliations.

Received: 1 October 2018 Accepted: 5 December 2018

Published online: 18 December 2018

\section{References}

1. Hanamura I, Huang Y, Zhan F, Barlogie B, Shaughnessy J. Prognostic value of cyclin D2 mRNA expression in newly diagnosed multiple myeloma treated with high-dose chemotherapy and tandem autologous stem cell transplantations. Leukemia. 2006;20:1288-90.

2. Woo YR, Kim JS, Lim JH, Hwang S, Kim M, Bae JM, Park YM, Min CK, Kim DW, Park HJ. Prevalence and clinicopathologic characteristics of multiple myeloma with cutaneous involvement: a ca se series from Korea. J Am Acad Dermatol. 2018;78(471-478):e474.

3. Bao ZJ, Liu X. Research progress on role of flow cytometry in diagnosis of multiple myeloma-review. Zhongguo Shi Yan Xue Ye Xue Za Zhi. 2016:24:1611-4.

4. Zang M, Zou D, Yu Z, Li F, Yi S, Ai X, Qin X, Feng X, Zhou W, Xu Y, et al. Detection of recurrent cytogenetic aberrations in multiple myeloma: a comparison between MLPA and iFI SH. Oncotarget. 2015;6:34276-87.

5. Pawlyn C, Morgan GJ. Evolutionary biology of high-risk multiple myeloma. Nat Rev Cancer. 2017:17:543-56.

6. Avet-Loiseau H, Durie BG, Cavo M, Attal M, Gutierrez N, Haessler J, Goldschmidt $\mathrm{H}$, Hajek R, Lee JH, Sezer $\mathrm{O}$, et al. Combining fluorescent in situ hybridization data with ISS staging improves risk assessment in myeloma: an International Myeloma Working Group collaborative project. Leukemia. 2013:27:711-7.

7. Palumbo A, Avet-Loiseau H, Oliva S, Lokhorst HM, Goldschmidt H, Rosinol L, Richardson P, Caltagirone S, Lahuerta JJ, Facon T, et al. Revised international staging system for multiple myeloma: a report from International Myeloma Working Group. J Clin Oncol. 2015:33:2863-9.

8. Weinhold N, Heuck CJ, Rosenthal A, Thanendrarajan S, Stein CK, Van Rhee F, Zangari M, Hoering A, Tian E, Davies FE, et al. Clinical value of molecular subtyping multiple myeloma using gene expression profiling. Leukemia. 2016:30:423-30.

9. Zhan F, Huang Y, Colla S, Stewart JP, Hanamura I, Gupta S, Epstein J, Yaccoby S, Sawyer J, Burington B, et al. The molecular classification of multiple myeloma. Blood. 2006;108:2020-8.

10. Paszekova H, Kryukov F, Kubiczkova L, Hajek R, Sevcikova S. High-risk multiple myeloma: different definitions, different outcomes? Clin Lymphoma Myeloma Leuk. 2014;14:24-30.

11. Marchesini M, Ogoti Y, Fiorini E, Aktas Samur A, Nezi L, D'Anca M, Storti P, Samur MK, Ganan-Gomez I, Fulciniti MT, et al. ILF2 is a regulator of RNA splicing and DNA damage response in 1q21-amplified multiple myeloma. Cancer Cell. 2017;32(88-100):e106.

12. Sun G, Cheng SY, Chen M, Lim CJ, Pallen CJ. Protein tyrosine phosphatase 'Á phosphotyrosyl-789 binds BCAR3 to position Cas for activation at integ rin-mediated focal adhesions. Mol Cell Biol. 2012;32:3776-89.

13. Wilson AL, Schrecengost RS, Guerrero MS, Thomas KS, Bouton AH. Breast cancer antiestrogen resistance 3 (BCAR3) promotes cell motility by regulating actin cytoskelet al and adhesion remodeling in invasive breast cancer cells. PLoS ONE. 2013:8:e65678.

14. Guo J, Canaff L, Rajadurai CV, Fils-Aimé N, Tian J, Dai M, Korah J, Villatoro M, Park M, Ali S, Lebrun JJ. Breast cancer anti-estrogen resistance 3 inhibits transforming growth factor $\beta / S$ mad signaling and associates with favorable breast cancer disease outcomes. Breast Cancer Res. 2014;16:476.

15. Oh MJ, van Agthoven T, Choi JE, Jeong YJ, Chung YH, Kim CM, Jhun BH. $B C A R 3$ regulates EGF-induced DNA synthesis in normal human breast MCF-12A cells. Biochem Biophys Res Commun. 2008:375:430-4. 
16. Cross AM, Wilson AL, Guerrero MS, Thomas KS, Bachir Al, Kubow KE, Horwitz AR, Bouton AH. Breast cancer antiestrogen resistance 3-p130Cas interactions promote adhesion disassembly and invasio $\mathrm{n}$ in breast cancer cells. Oncogene. 2016;35:5850-9.

17. Vanden Borre P, Near RI, Makkinje A, Mostoslavsky G, Lerner A. BCAR3/ AND-34 can signal independent of complex formation with CAS family members or the presence of p 130Cas. Cell Signal. 2011;23:1030-40.

18. Makkinje A, Vanden Borre P, Near RI, Patel PS, Lerner A. Breast cancer anti-estrogen resistance 3 (BCAR3) protein augments binding of the c-Src SH3 domain to Crk-associated substrate (p130cas). J Biol Chem. 2012;287:27703-14

19. Schrecengost RS, Riggins RB, Thomas KS, Guerrero MS, Bouton AH. Breast cancer antiestrogen resistance-3 expression regulates breast cancer cell migration through pro motion of p130Cas membrane localization and membrane ruffling. Cancer Res. 2007;67:6174-82.

20. Pennekamp P, Feldner S, Seesing FJ, Psathaki OE, Schöler HR, Wieacker P, Dworniczak B. Bcar3 is expressed in sertoli cells and germ cells of the developing testis in mice. Sex Dev. 2011;5(4):197-204.

21. Kondo T, Nakamori T, Nagai H, Takeshita A, Kusakabe KT, Okada T. A novel spontaneous mutation of BCAR3 results in extrusion cataracts in CF\#1 mouse strain. Mamm Genome. 2016;27:451-9.

22. Zhou K, Diebel KW, Holy J, Skildum A, Odean E, Hicks DA, Schotl B, Abrahante JE, Spillman MA, Bemis LT. A tRNA fragment, tRF5-Glu, regulates BCAR3 expression and proliferation in ovarian cancer cells. Oncotarget. 2017:8:95377-91.

23. Shi L, Campbell G, Jones WD, Campagne F, Wen Z, Walker SJ, Su Z, Chu TM, Goodsaid FM, Pusztai L, et al. The MicroArray quality control (MAQC)II study of common practices for the development and validation of microarray-based predictive models. Nat Biotechnol. 2010;28:827-38.

24. Weinhold N, Ashby C, Rasche L, Chavan SS, Stein C, Stephens OW, Tytarenko R, Bauer MA, Meissner T, Deshpande S, et al. Clonal selection and double-hit events involving tumor suppressor genes underlie relapse in myeloma. Blood. 2016;128:1735-44.

25. Broyl A, Hose D, Lokhorst H, de Knegt Y, Peeters J, Jauch A, Bertsch U, Buijs A, Stevens-Kroef M, Beverloo HB, et al. Gene expression profiling for molecular classification of multiple myeloma in newly diagnosed patient s. Blood. 2010;116:2543-53.

26. Miannay B, Minvielle S, Roux O, Drouin P, Avet-Loiseau H, Guérin-Charbonnel C, Gouraud W, Attal M, Facon T, Munshi NC, et al. Logic programming reveals alteration of key transcription factors in multiple myeloma. Sci Rep. 2017;7:9257.

27. Mulligan G, Mitsiades C, Bryant B, Zhan F, Chng WJ, Roels S, Koenig E, Fergus A, Huang Y, Richardson P, et al. Gene expression profiling and correlation with outcome in clinical trials of the proteasome inhibitor bortezomib. Blood. 2007;109:3177-88.

28. Chauhan D, Tian Z, Nicholson B, Kumar KG, Zhou B, Carrasco R, McDermott JL, Leach CA, Fulcinniti M, Kodrasov MP, et al. A small molecule inhibitor of ubiquitin-specific protease-7 induces apoptosis in multiple myeloma cel Is and overcomes bortezomib resistance. Cancer Cell. 2012;22:345-58.

29. Zhou W, Yang Y, Xia J, Wang H, Salama ME, Xiong W, Xu H, Shetty S, Chen T, Zeng $Z$, et al. NEK2 induces drug resistance mainly through activation of efflux drug pumps and is associated with po or prognosis in myeloma and other cancers. Cancer Cell. 2013;23:48-62.
30. Huang DW, Sherman BT, Lempicki RA. Systematic and integrative analysis of large gene lists using DAVID bioinformatics resources. Nat Protoc. 2009;4:44-57.

31. Child JA, Morgan GJ, Davies FE, Owen RG, Bell SE, Hawkins K, Brown J, Drayson MT, Selby PJ. Medical Research Council Adult Leukaemia Working P: high-dose chemotherapy with hematopoietic stem-cell rescue for multiple myeloma. N Engl J Med. 2003;348:1875-83.

32. Esma F, Salvini M, Troia R, Boccadoro M, Larocca A, Pautasso C. Melphalan hydrochloride for the treatment of multiple myeloma. Expert Opin Pharmacother. 2017;18:1127-36.

33. Landgren $\mathrm{O}$, Waxman AJ. Multiple myeloma precursor disease. JAMA. 2010;304:2397-404

34. Stone MJ, Lieberman ZH, Chakmakjian ZH, Matthews JL. Coexistent multiple myeloma and primary hyperparathyroidism. JAMA. 1982;247:823-4.

35. Bianchi G, Anderson KC. Understanding biology to tackle the disease: multiple myeloma from bench to bedside, and back. CA Cancer J Clin. 2014;64:422-44.

36. Kumar SK, Rajkumar V, Kyle RA, van Duin M, Sonneveld P, Mateos MV, Gay F, Anderson KC. Multiple myeloma. Nat Rev Dis Primers. 2017;3:17046.

37. Cai D, lyer A, Felekkis KN, Near Rl, Luo Z, Chernoff J, Albanese C, Pestell RG, Lerner A. AND-34/BCAR3, a GDP exchange factor whose overexpression confers antiestrogen resistance, activates $\mathrm{R}$ ac, PAK1, and the cyclin D1 promoter. Cancer Res. 2003;63:6802-8.

38. van Agthoven T, van Agthoven TL, Dekker A, van der Spek PJ, Vreede $L$, Dorssers LC. Identification of BCAR3 by a random search for genes involved in antiestrogen resistance of human bre ast cancer cells. EMBO J. 1998; 17:2799-808.

39. Williams SA, Harata-Lee Y, Comerford I, Anderson RL, Smyth MJ, McColl SR. Multiple functions of CXCL12 in a syngeneic model of breast cancer. Mol Cancer. 2010;9:250.

40. Attal M, Harousseau JL, Stoppa AM, Sotto JJ, Fuzibet JG, Rossi JF, Casassus $\mathrm{P}$, Maisonneuve $\mathrm{H}$, Facon T, Ifrah N, Payen C. A prospective, randomized trial of autologous bone marrow transplantation and chemotherapy in multiple myeloma. N Engl J Med. 1996;335(2):91-7.

41. Gooley TA, Chien JW, Pergam SA, Hingorani S, Sorror ML, Boeckh M, Martin PJ, Sandmaier BM, Marr KA, Appelbaum FR, et al. Reduced mortality after allogeneic hematopoietic-cell transplantation. N Engl J Med. 2010;363:2091-101.

42. Chepovetsky J, Chari A, Jagannath S, Ramanthan L. A case of IgD myeloma representing a missed biclonal paraproteinemia or isotype switch in a patient $\mathrm{p}$ reviously diagnosed with IgA-lambda myeloma. Clin Chim Acta. 2013;425:233-5.

43. Suehara Y, Takamatsu H, Fukumoto K, Fujisawa M, Narita K, Usui Y, Takeuchi M, Endean K, Matsue K. Abnormal heavy/light chain ratio after treatment is associated with shorter survival in patients with $\lg A$ myeloma. Cancer Sci. 2017;108:187-92.

\footnotetext{
Ready to submit your research? Choose BMC and benefit from:

- fast, convenient online submission

- thorough peer review by experienced researchers in your field

- rapid publication on acceptance

- support for research data, including large and complex data types

- gold Open Access which fosters wider collaboration and increased citations

- maximum visibility for your research: over $100 \mathrm{M}$ website views per year
}

At BMC, research is always in progress.

Learn more biomedcentral.com/submissions 